\title{
ON THE FORMATION OF ELLIPTICAL GALAXIES
}

\author{
J. R. GOTT III
}

California Institute of Technology, Dept. of Astronomy, Pasadena, Calif., U.S.A.

\begin{abstract}
Simple collapse and violent relaxation models produce sharply cut off envelopes with $\varrho(r) \propto r^{-4}$, whereas actual elliptical galaxies have more extended envelopes approximately given by $\varrho(r) \propto r^{-3}$ as implied by Hubble's Law. Numerical models are presented, showing that when cosmological infall effects are included, galaxies are produced with more extended envelopes having a $\varrho(r) \propto r^{-2.8}$ dependence in excellent agreement with Hubble's Law. We have also computed a realistic rotating model of an E5 galaxy which includes infall effects and where the angular momentum of the galaxy is gained through tidal interactions with neighboring protogalaxies. The envelope displays a Hubble dependence but at great distances shows a cutoff due to the tidal effects. The model is compared to the E5 galaxy NGC 4697 and provides an outstandingly good fit to the observations. Except for simple scaling, this excellent fit is produced without the use of any free fitting parameters.
\end{abstract}

\section{Introduction}

I would like to present a different point of view, namely that elliptical galaxies are produced by a dissipationless collapse and relaxation process.* According to current cosmological theories galaxies begin as small density perturbations present at the epoch of hydrogen recombination at a redshift of $z \sim 1000$. Because of the excess density in the perturbation the density in the protogalaxy exceeds the critical density $\left[\varrho>\varrho_{c i}=3 H_{i}^{2} / 8 \pi G\right]$ and is therefore gravitationally bound. The protogalaxy initially is expanding with the uniform Hubble expansion $\mathbf{V}=H_{i} \mathbf{r}$, but because it is gravitationally bound, it will expand to reach a maximum radius, stop momentarily and then begin to collapse. If star formation in the early protogalaxy [perhaps occurring in early globular clusters as proposed by Peebles and Dicke (1968)] is sufficiently rapid so that star formation is essentially complete by the time it reaches the point of maximum collapse, then one has a dissipationless collapse and relaxation process.

The dissipationless collapse and relaxation process for a stellar system offers several attractive features in explaining elliptical galaxies. First, the Lynden-Bell (1967) violent relaxation process is expected to operate and lead naturally to the relaxed elliptical isophotes observed in elliptical galaxies. In such numerical models (Gott, 1973) the equilibrium structure is reached after only a couple of free fall times and the isophotes of the equilibrium galaxy are indeed nicely elliptical. Secondly, the dissipationless collapse picture explains in a natural way the observed flattenings of elliptical galaxies.

Consider a nonrotating sphere of stars with no initial kinetic energy and a radius of $R_{0}$, corresponding to the point of maximum expansion. Let $E_{b}$ be the total binding energy which is conserved in the dissipationless collapse. Then initially $-E_{b}=T+W$ with $W=-\left(3 G M_{\mathrm{gal}}^{2} / 5 R_{0}\right)=-E_{b}$ and $T=0$. Let it collapse and undergo violent

* The results presented here will be discussed in greater detail in a forthcoming paper, Gott (1975). 
relaxation. When virial equilibrium is reached, $2 T+W=0$ and $-E_{b}=T+W$ so $W=-2 E_{b}$, or twice its original value, and the equilibrium system will be spherical with a characteristic radius of $\sim \frac{1}{2} R_{0}$. Now consider a rotating galaxy of radius $R_{0}$ and $\Omega=\Omega_{0}$ so that it is initially holding itself up against gravity in the equatorial plane. This is surely an upper limit on the amount of rotation we would expect cosmologically, and is an amount which can be produced from tidal interactions with neighboring protogalaxies as we shall see below. Let this galaxy collapse. It continues holding itself up in the equatorial plane, leaving $r \cong R_{0}$ in that direction. Rotation does not inhibit collapse perpendicular to the plane, so to first approximation the collapse proceeds perpendicularly as in the spherical case to give $r=\frac{1}{2} R_{0}$. These approximations indicate an equilibrium galaxy with a 2 to 1 axial ratio (i.e., an E5 galaxy). Thus, with a dissipationless collapse picture we expect elliptical galaxies to show intrinsic flattenings approximately in the range E0-E5. Significantly, elliptical galaxies are observed to have intrinsic flattenings in the range E0-E5 with just a few E6 galaxies and no E7 or flatter galaxies. The fact that elliptical galaxies have intrinsic flattenings in just the range predicted by the dissipationless picture is strong evidence for this mechanism. When gas is present, as in spiral galaxies, it is quite dissipative forming disk systems with characteristic flattenings of 10 or even 20 to 1 .

The simple models of the collapse and relaxation of a spherical cloud of stars thus give equilibrium models having many of the properties of elliptical galaxies. The main difficulty with this picture is that the simple models considered in Gott (1973) produce elliptical galaxies with envelopes in which the density falls off according to $\varrho(r) \propto r^{-4}$. Observed elliptical galaxies have envelopes that are much more extended than this. The envelopes of elliptical galaxies can be modeled well by Hubble's Law (1930), which gives a projected surface density of the form $I(r)=I_{0} /[1+(r / a)]^{2}$ where $a$ is a constant. This requires $I(r) \propto r^{-2}$ in the envelope and thus $\varrho(r) \propto r^{-3}$. Other $\mathrm{N}$-body calculations that study the collapse and relaxation of a spherical cloud of stars show the same problem. Models by Peebles (1970), Hénon (1964), and Aarseth (1973) all produce sharply cut off envelopes with the $r^{-4}$ dependence. In the present paper we will show that the simple models ignore cosmological infall effects and that when these effects are included we can explain the main features of elliptical galaxy envelopes.

\section{Cosmological Infall}

The dynamics of cosmological infall have been discussed in detail in relation to infall into clusters of galaxies by Gott and Gunn (1971) and Gunn and Gott (1972). We shall present here just the primary results needed for elliptical galaxies. In accordance with the standard big bang cosmology we envision the protogalaxy beginning as an isothermal density perturbation present at the epoch of recombination at redshift $z_{i} \sim 1000$. Initially there is a uniform Hubble flow $\left(\mathbf{V}=H_{i} \mathbf{r}\right)$. Because of the excess density, the material in the protogalaxy is gravitationally bound. Its expansion is slowed, so the perturbation reaches a maximum radius and begins to collapse, com- 
pleting the collapse at time $T_{c}$. Material outside the perturbation proper can also be gravitationally bound and suffers infall into the galaxy at $t>T_{c}$.

We assume that at the epoch of recombination there exists a spherical region of radius $R_{i}$ which has uniform density slightly higher than the surrounding region. Such a perturbation structure is in the spirit of a white noise spectrum of density fluctuations (i.e., Poisson distribution of points) as studied by Press and Schechter (1974) and Peebles (1974). In such a white noise situation the density in the region just outside a known positive density fluctuation is, on the average, just equal to the average density. Press and Schechter (1974), using this model and standard dynamics, were able to derive a theoretical luminosity function for galaxies in good agreement with the observations. Thus, we adopt the condition that the density outside the perturbation proper should be of average density. Let $\varrho_{c i}$ be the critical density at the epoch $z_{i}=1000$

$$
\varrho_{c i}=\frac{3 H_{i}^{2}}{8 \pi G} \text {. }
$$

For densities less than the critical density the expansion is unbounded; for densities in excess of $\varrho_{c i}$ the material will expand to a maximum radius and collapse. Let $\varrho_{e i}$ be the external density which we set equal to the mean density in the universe at epoch $z_{i}$. From Gunn and Gott (1972) one can show that

$$
\varrho_{e i}=\varrho_{c i} \cdot \frac{2 q_{0}\left(1+z_{i}\right)}{\left(1-2 q_{0}\right)+2 q_{0}\left(1+z_{i}\right)} .
$$

We consider pressureless cosmologies with no cosmological constant, so the deceleration parameter $q_{0}$ is given by

$$
3 H_{0}^{2} q_{0}=4 \pi G \varrho_{0},
$$

where the subscript ' 0 ' refers to the present. If $q_{0}$ is greater than $\frac{1}{2}$ the universe is closed; if $0<q_{0}<\frac{1}{2}$, the universe is open with unbounded expansion. Let $\varrho_{i}$ be the density in the perturbation proper $r<R_{i}$ and define $\varrho_{+}=\varrho_{i}-\varrho_{c i}$. To an accuracy of several percent for small $\varrho_{+}$relative to $\varrho_{c i}$ we find

$$
T_{c} \simeq \frac{\pi}{H_{i}}\left(\frac{\varrho_{c i}}{\varrho_{+}}\right)^{3 / 2},
$$

where $T_{c}$ is just the age of the universe when the perturbation completes its collapse. The perturbation proper is of uniform density initially and remains of uniform density throughout its expansion and collapse. The initial perturbation has a mass $M_{0} \simeq 4 \pi \varrho_{c i} R_{i}^{3} / 3$. One can show (Gunn and Gott, 1972) that for $t>T_{c}$ the mass of the galaxy including infall is given by

$$
\frac{M(t)}{M_{0}}=1+\frac{\left(t / T_{c}\right)^{2 / 3}-1}{1+\left(1-2 q_{0}\right)\left(\frac{H_{0} t}{2 \pi q_{0}}\right)^{2 / 3}} .
$$


With $q_{0}=\frac{1}{2}, M(t)=M_{0} \cdot\left(t / T_{c}\right)^{2 / 3}$ and the infall material can be considerable. This infall material is less tightly bound and should produce a more extended envelope. As we shall see below this effect is precisely sufficient to produce elliptical galaxy models in agreement with the observations.

From Equation (5) it is clear that the infall depends on the value of $q_{0}$. However, for $t \ll H_{0}^{-1}$ it is clear that in all cosmologies the infall approximates closely that of the $q_{0}=0.5$ case. This is because densities in all cosmological models approach asymptotically the critical density at early epochs as indicated by Equation (2). In particular the model we will compare with NGC 4697 has a collapse time for the central perturbation of $T_{c} \sim 3.3 \times 10^{7} \mathrm{yr}$, which combined with $H_{0}=55 \mathrm{~km} \mathrm{~s}^{-1} \mathrm{Mpc}^{-1}$ gives $H_{0} T_{c}=0.0019$ so even if $q_{0}$ is as low as $q_{0}=0.02$ (see Gott et al., 1974) Equation (5) shows that for times shortly after $t=T_{c}$ the infall is within about $6 \%$ of that predicted by the $q_{0}=\frac{1}{2}$ model. Thus, the collapse times of elliptical galaxies are typically short enough so that the infall and resulting envelope structure are approximated well by the $q_{0}=\frac{1}{2}$ model. This is significant not only because it makes the results essentially independent of the cosmological model but because it produces ellipticals with essentially the same envelope structure relatively independently of their surroundings. If the open cosmological picture of Gott et al. (1974) is correct then ellipticals in the field see a local cosmology with $q_{0}=0.025$, while ellipticals in bound clusters experience an effective $q_{0}>\frac{1}{2}$. The above results indicate that these differences will not result in great differences in infall structure.

\section{Spherical Systems}

Two spherical models are computed using 1000 stars with a variant of Hénon's spherical shell technique. Model I is the simple collapse of a uniform density sphere of stars with no infall. This model is thus representative of the class of simple models like those of Hénon (1964), Peebles (1970), and Gott (1973). The calculation is started at the point of maximum expansion.

Model II includes the effects of cosmological infall. As discussed in Section 2 it is sufficient to consider a single case with $q_{0}=\frac{1}{2}$. For the initial perturbation $r<R_{i}$ we adopt $\varrho=\varrho_{c i}+\varrho_{+}$such that $T_{c}$ computed by Equation $(6)$ is $T_{c}=\pi\left(R_{0}^{3} / 2 G M_{0}\right)^{1 / 2}$ matching the $T_{c}$ of model I. For $r>R_{i}$ we adopt $\varrho=\varrho_{c i}$. The initial conditions are set at the epoch of recombination at $z=1000$; one can easily show that the results are independent of this particular epoch as long as $\varrho_{+}$is chosen so as to yield the correct $T_{c}$ and $H_{i} \ll T_{c}$. We assign 100 stars of mass $0.01 M_{0}$ each to the initial perturbation and 900 stars of mass $0.01 M_{0}$ to the adjacent surrounding volume. The trajectory of each star is computed analytically via the formulas in Gunn and Gott (1972) up until $t=\frac{1}{2} T_{c}$, when the calculation is continued numerically using the shell technique mentioned above.

We note that these elliptical galaxies have enough stars so as to be essentially collisionless systems (i.e., the two-body relaxation time is significantly longer than the times over which the system is studied). We have made several checks to assure 
that our systems are indeed essentially collisionless (see Gott, 1975), and that the only significant relaxation is the Lynden-Bell violent relaxation.

The model I results are quite similar to those found in Gott (1973). By time $t=\frac{3}{2} T_{c}$ the system had reached its equilibrium structure. The model I results displayed in Figures 1 and 2 are for the equilibrium structure and refer to time averages over the period $t=2 T_{c}$ to $3 T_{c}$.

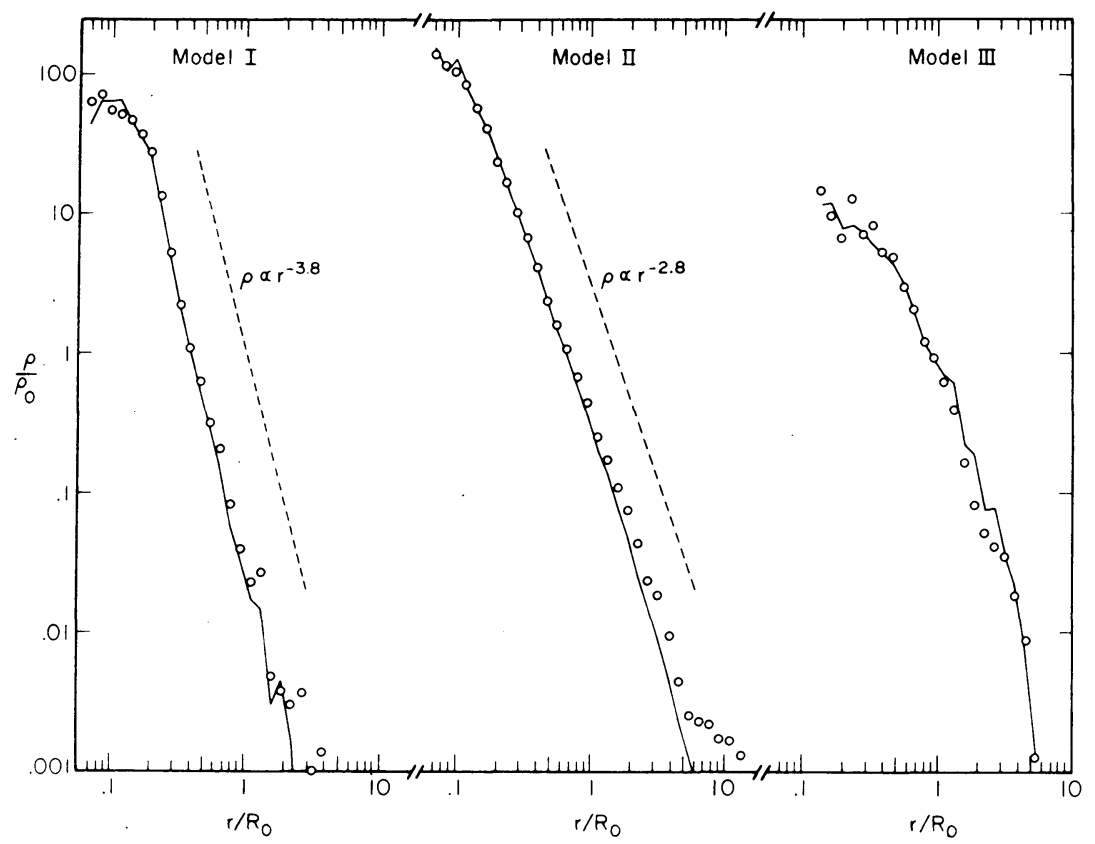

Fig. 1. Stellar density in the galactic plane as a function of radius for the equilibrium models. The open circles represent the actual runs of density with radius while the solid lines represent that expected from satisfaction of the stationary collisionless Boltzmann equation. The density $\varrho_{0}=3 M_{0} / 4 \pi R_{0}^{3}$ is a standard density which is the same for each of the models. Model I is a standard spherical collapse model without infall, model II includes cosmological infall.and thus has a much more extended envelope. The dotted lines give the best power law fit to each of the envelopes. Model II with $\varrho \propto r^{-2.8}$ agrees closely with $\varrho \propto r^{-3}$ predicted from satisfaction of Hubble's Law $I=I_{0} /[1+(r / a)]^{2}$. Model III is a rotating model whose rotation has been derived through tidal interactions with neighboring protogalaxies. It includes both cosmological infall effects and tidal interaction effects.

In Figure 1 the run of density versus radius is presented. The solid line represents the expected run of density expected from satisfaction of the stationary collisionless Boltzmann equation.

$$
\begin{aligned}
-\frac{\partial \Phi}{\partial r}=-\frac{\left\langle V_{\phi}^{2}\right\rangle}{r} & +\left\langle V_{r}^{2}\right\rangle\left[\frac{\partial \ln \varrho}{\partial r}+\frac{\partial \ln \left\langle V_{r}^{2}\right\rangle}{\partial r}+\frac{1}{r}\left(1-\frac{\left\langle V_{\theta}^{2}\right\rangle}{\left\langle V_{r}^{2}\right\rangle}\right)+\right. \\
& \left.+\frac{1}{r}\left(1-\frac{\left\langle\left(V_{\phi}-\left\langle V_{\phi}\right\rangle\right)^{2}\right\rangle}{\left\langle V_{r}^{2}\right\rangle}\right)\right] .
\end{aligned}
$$

There is excellent agreement between the observed density and the solid line showing 
that the system is essentially collisionless and that it has reached an equilibrium structure. In the envelope, the best fit is given by $\varrho \propto r^{-3.8}$ as indicated by the dashed line in Figure 1.

In model II the infall, given by $M(t)=M_{0} \cdot\left(t / T_{c}\right)^{2 / 3}$, goes as a power law with the infall rate depending only on the mass accumulated up to that time. Thus for $t \gg T_{c}$ there are no intrinsic scales to the problem. The solution is what Press and Schechter (1974) would refer to as selfsimilar. With no intrinsic scale present, it is easy to show that the expected envelope structure should be a power law in the density. A numerical experiment is then needed to determine the slope of this power law. An important factor is the relaxation of each shell of stars as it suffers infall. The results presented in Figure 1 show that the equilibrium envelope of model II has indeed a remarkably straight power law dependence with $\varrho \propto r^{-2.8}$ in virtually exact agreement with the Hubble's Law envelope dependence $\varrho \propto r^{-3}$. Model II satisfies the stationary collisionless Boltzmann equation, showing that it has reached a stationary equilibrium configuration. For $r>5 R_{0}$ there is a sharp departure from satisfaction of the stationary collisionless Boltzmann equation. This is due to the fact that the outer shells of stars have not yet suffered infall and are still traveling outward. The outermost shell of stars at radius $r=14 R_{0}$ has a Hubble expansion that has been only slightly slowed. The run of density with radius presented in Figure 1 is taken

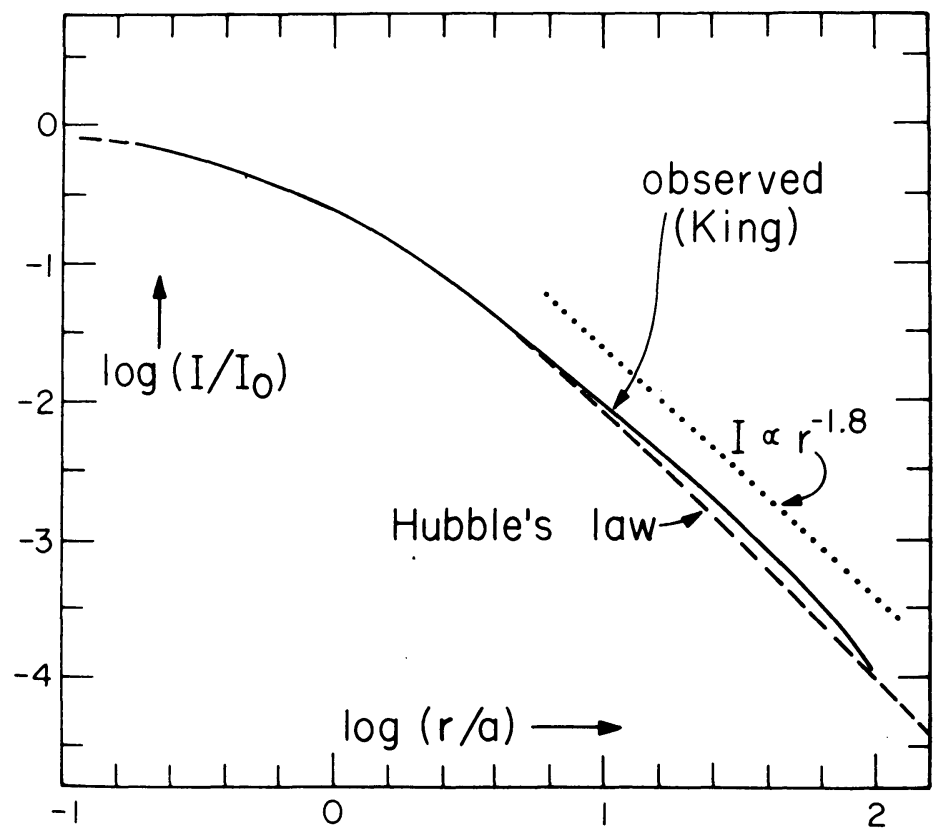

Fig. 2. Surface brightness as a function of projected radius. The solid line represents an average of 13 observed galaxies for which King has done accurate photometry. For comparison the dashed curve gives Hubble's Law $I=I_{0} /[1+(r / a)]^{2}$. The dotted curve $I \propto r^{-1.8}$ gives the slope expected for an envelope where $\varrho \propto r^{-2.8}$ as in model II. Thus model II, which includes infall, gives an envelope structure in good agreement with the observations. 
at time $t \sim 6 T_{c}$ (i.e., a time average from 5.5 to $6.5 T_{c}$ ). As time goes on, the background cosmological density continues to go down, and more and more of the envelope separates out continuing to show the same power law dependence.

Let us compare the results for model II with those for observed elliptical galaxies. In Figure 2 is plotted a mean observed curve of light intensity (flux $\operatorname{arcsec}^{-2}$ ) vs radius for 13 individual galaxies from photometry obtained by King (private communication). The curves for the individual galaxies have been scaled both in central intensity and radius. For comparison, the mean observed curve has been fit (Wilson, 1973) with the best fit of Hubble's Law. The radial distances are measured in terms of the constant $a$ in Hubble's Law. The fit of Hubble's Law to the observed data is certainly outstanding, particularly in the central regions and the fit in the envelope is also quite close. It is noteworthy also that the curves of $I(r)$ for the 13 individual galaxies all lie quite close to the mean curve, differing from it less in all cases than the maximum difference between the mean curve and Hubble's Law. This is one of the extraordinary properties of elliptical galaxies, that they exhibit envelopes with nearly identical radial structures. Model II, which includes infall, predicts $\varrho(r) \propto r^{-2.8}$ and $I(r) \propto r^{-1.8}$. For comparison we have drawn a dotted line showing the slope $I \propto r^{-1.8}$. It can be seen that this slope is in excellent agreement with the data over the decade and a half in radius of interest. Thus the infall picture provides a ready explanation for the observed density falloff in the envelopes of elliptical galaxies.

\section{Rotating Systems}

\subsection{INTRODUCTION}

Peebles (1971) proposes that galaxies acquire their angular momentum through tidal interactions. The original protogalaxy is undoubtedly irregular in shape and thus the tidal action of the neighboring protogalaxies exerts a torque on it. Peebles (1971) has shown by numerical experiments that the angular momentum transfer is accomplished primarily around the time the protogalaxy reaches its point of maximum expansion. He also shows that the maximum amount of angular momentum acquired by the protogalaxy is (to a factor of order unity) $J=(2 / 5) M_{0} R_{0}^{2} \Omega_{0}$ where $\Omega_{0}=$ $=\left(G M_{0} / R_{0}^{3}\right)^{1 / 2}$ is the angular velocity required to just prevent collapse in the equatorial plane. By application of simple virial theorem arguments earlier it was shown that the maximally rotating galaxy $\left(\Omega=\Omega_{0}\right)$ is left with a flattening of at most 2 to 1 , i.e., an E5 galaxy. Thus, it was argued that one should expect to see elliptical galaxies in the range E0 to approximately E5, which agrees quite well with the observations.

\subsection{MeThOD AND INITIAL CONDITIONS}

We wish to study the collapse and relaxation of a protogalaxy which has been set into rotation by tidal interactions and where infall effects are included. We first define a spherical coordinate system $(r, \theta, \phi)$ centered on the protogalaxy and having an axis $(\theta=0)$ equal to the proposed rotation axis of the protogalaxy. We start with 2000 stars, 200 stars for the initial perturbation and 1800 stars in the outlying infall region 
(so $m_{s}=0.005 M_{0}$ ). We start by giving the stars radial positions and velocities at $t=\frac{1}{2} T_{c}$ just as done for model II. We give the galaxy an irregular shape by confining the stars to $0<\phi<\pi / 2$ and $\pi<\phi<\frac{3}{2} \pi$. Thus, at the point of maximum expansion the initial perturbation has density $\varrho=2 \varrho_{0}$ in two opposite quadrants and density $\varrho=0$ in the other two quadrants. Likewise the infall material is confined to these quadrants.

The tidal effects of neighboring protogalaxies are effective for only a short time near $t=\frac{1}{2} T_{c}$ (assuming that all the protogalaxies have similar collapse times). At earlier times the other protogalaxies are just small density fluctuations $\delta \varrho / \varrho \ll 1$ and Peebles' (1971) calculations show the angular momentum transfer to be small. By $t=\frac{1}{2} T_{c}$ the protogalaxies have separated out and are behaving essentially as point masses but as time goes on their tidal effects decrease since the distances between the protogalaxies are growing as $d \propto t^{2 / 3}$ and the tidal force goes as $d^{-3}$. Thus, as Peebles' calculations show, the angular momentum transfer occurs primarily around $t=\frac{1}{2} T_{c}$. Our initial conditions are taken to start at $t=\frac{1}{2} T_{c}$ so we adopt the following simple model for the tidal interactions of the neighboring protogalaxies. We define the perturbing potential

$$
\Phi_{\text {tidal }}=-2\left(G M / d^{3}\right)\left(x^{2}\right),
$$

where $x=r \sin \theta \cos \phi$. One may think of this perturbing potential arising from the action of two protogalaxies of mass $M$ and distance $d$ on either side of the galaxy in question (i.e., at $\theta=\pi / 2, \phi=0$ and $\theta=\pi / 2, \phi=\pi$ ). We have used for simplicity here just the lowest order spherical harmonic. The perturbing potential should act for a time $\Delta t \sim T_{c}$ approximately centered on $t=\frac{1}{2} T_{c}$. Thus each star should receive a kick in velocity due to the tidal forces given by

$$
\Delta \mathbf{V}(r, \theta, \phi) \simeq \Delta t \cdot \nabla \Phi_{\text {tidal }}
$$

We thus compute the velocity kick given each star due to the tidal forces and add that extra velocity to the stars original velocity as defined from the initial conditions of model II. Since $\Delta t$ is assumed to be short we will not alter the positions of the stars. Since the tidal forces die out rapidly after $t \sim \frac{1}{2} T_{c}$ we will assume their entire effect is represented by the velocity kicks and set the disturbing potential equal to zero after $t=\frac{1}{2} T_{c}$. Because of the irregular shape of the protogalaxy with the stars confined to opposite quadrants, it is easy to show that a net angular momentum is imparted to the protogalaxy. The value of the tidal potential is set, see Equation (7) at a value $\left(M / d^{3}\right)=0.25\left(M_{0} / R_{0}^{3}\right)$. This value is sufficient to produce an E5 galaxy, as we shall see. The value above was chosen on the basis that it would produce a reasonable amount of rotation. In Gott (1975) we compare this value with what one would calculate a priori given a random distribution of neighboring protogalaxies similar to the one we are considering.

The result of this calculation gives a root mean square tidal torque corresponding to $\left(M / d^{3}\right)=0.15\left(M_{0} / R_{0}^{3}\right)$. Considering that Peebles $(1969,1971)$ estimates that such calculations are accurate to no more than a factor of 4 , the above value is in excellent 
agreement with the value $\left(M / d^{3}\right)=0.25\left(M_{0} / R_{0}^{3}\right)$ adopted for model III on the basis of producing the required amount of rotation. The present picture is that all protogalaxies experience an rms tidal field due to neighboring protogalaxies; when the irregular shape of the protogalaxy is aligned properly with the tidal field (as is model III) the maximum net torque is applied and a maximally rotating galaxy is produced. Since model III produces an E5 galaxy it is clear that production of elliptical galaxies in the range E0 to E5 is consistent with the tidal interaction hypothesis.

With the initial conditions as given, the collapse will be non-axisymmetric and individual stars, seeing a non-axisymmetric potential, will either gain or lose angular momentum. Unfortunately such a three dimensional calculation is beyond the range of present computers. Instead we simulate the mixing of angular momenta produced by the violent relaxation by giving the stars extra random velocities in the model II initial conditions and then fix the system to be axisymmetric. The non-axisymmetric relaxation phase, mimicked by the extra random velocities, should lead to an axisymmetric final state so our approximation of an axisymmetric potential is reasonable.

The collapse and violent relaxation phase can now be followed by the axisymmetric potential method of Gott (1973).

\subsection{Results}

The system was allowed to collapse and relax and the central region reached an equilibrium configuration by $t=2 T_{c}$ (where all times are measured from the beginning of the universe). The envelope infall continued after this. The outer portions of the envelope have escape energy and are lost from the galaxy, i.e., stolen away by the neighboring protogalaxies. The infall is thus cut off and the envelope settles down by about $t=10 T_{c}$. We take a time average over $10.5 T_{c}<t<14.5 T_{c}$ for the following data.

From Figure 1 it can be seen that the equilibrium galaxy (model III) closely satisfies the stationary collisionless Boltzmann equation. The envelope is extended like that of model II due to the infall, the envelope retaining the basic $\varrho \propto r^{-2.8}$ dependence but the tidal effects introducing a cutoff at large radii.

We wish to compare the results of model III with the best studied E5 galaxy NGC 4697. The fact that there are no observed elliptical galaxies appreciably flatter than E5 means that the galaxy must be seen essentially edge on and projection effects need not be considered. Figure 3 shows the model III data compared with the actual run of $I(r)$ obtained by King (private communication) for NGC 4697. The fit is certainly remarkably good. It is important to remember that, except for simple scaling, no free fitting parameters have been used to achieve this fit. (We have made only one rotating model. Started with reasonable initial conditions, it is compared with the best studied galaxy of its type. Thus, there has been no adjustment of the model parameters to improve the fit.) Figure 4 shows a comparison of the runs of ellipticity with radius. The scale has been set already by the intensity vs radius fit of Figure 3, so even the scale in Figure 4 is not adjustable. The data for model III is more scattered here due 


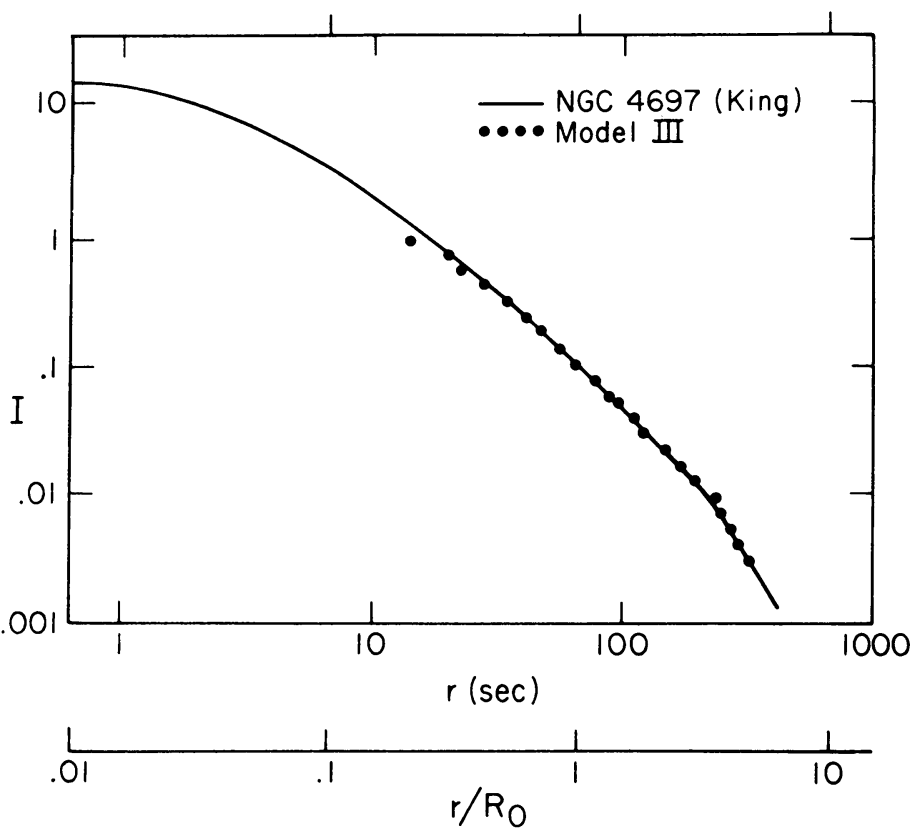

Fig. 3. A comparison of the results of model III and NGC 4697. The solid line plots surface brightness vs projected radius for NGC 4697 as obtained by King (private communication). The dots show similar surface brightness vs projected radius data for model III. It is noteworthy that except for simple scaling this excellent fit is obtained without the use of any free fitting parameters.

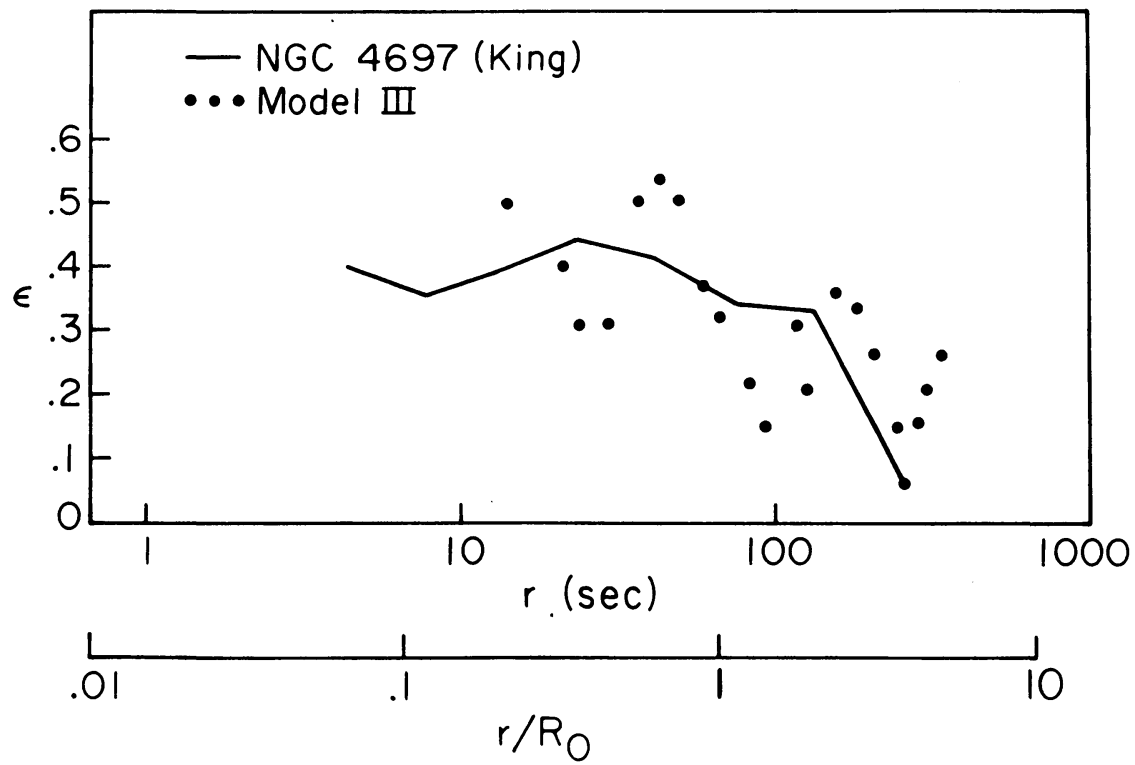

Fig. 4. A comparison of ellipticity $\varepsilon=(a-b) / a$ vs radius in model III and NGC 4697 . The two radial scales are preset by the fit of Figure 3. Although the model III data contain considerable statistical scatter. it is clear that in the mean it closely parallels the data from NGC 4697. 
to the unavoidably small number of stars in the envelope. However, given this random scatter, again the fit is quite good.

\subsection{DisCUSSION}

Wilson (1973) by bringing together considerable photometric data by King has shown that (1) elliptical galaxies regardless of intrinsic flattening have curves of intensity versus radius $[I(r)]$ that are remarkably similar (i.e., Figure 2);(2) curves of ellipticity versus radius $[\varepsilon(r)]$ show no particular pattern; in some cases the ellipticity increases, then decreases, in some it starts high and decreases, and in some it increases steadily outward. The present theory accounts easily for these two remarkable facts. From the previous sections it can be seen that the radial structure of the galaxy is determined virtually entirely by the infall effects and the tidal interactions which are similar for all protogalaxies. The ellipticity of the galaxy on the other hand is determined by the degree of irregularity of the original protogalaxy. If the original protogalaxy is spherically symmetric, then a spherical equilibrium galaxy is produced; if the original protogalaxy is irregular in shape (like model III where stars are confined to opposite quadrants) then the tidal action of neighboring protogalaxies can produce a rotating galaxy. The radial structure which is determined effectively by the binding energies of the different stars is unaffected by the irregularities in shape ( $\phi, \theta$ distribution) and depends through infall and tidal action primarily on the radial distribution. So Wilson's result number (1) is to be expected. It is reasonable that the irregularity in shape may be a function of radius. If the perturbation proper is spherical but the infall material is confined to quadrants, we will produce a galaxy with nearly circular isophotes near the center and more elliptical isophotes in the envelope. On the other hand, if the initial perturbation is irregular and the infall is spherically symmetric, one would have a galaxy with ellipticity decreasing outwards. Combine these effects with projection effects and it is clear that a variety of runs of eccentricity versus radius can be produced in agreement with Wilson's result (2). Despite the fact that a variety of runs of ellipticity are possible the runs of ellipticity for model III and NGC 4697 are still in good agreement. Two factors make this result plausible. First, an E5 galaxy represents the maximum possible flattening, so it is reasonable that the irregularities in shape might be of unit strength both in the perturbation proper and the envelope as well (as in model III). Secondly, since it is E5, we know we are seeing the galaxy virtually edge on (as assumed for model III) so there are no projection effects.

\section{Conclusions}

In the present paper we present detailed models for the formation of elliptical galaxies via a dissipationless collapse and violent relaxation process with cosmological infall effects included.

It is relevant to contrast this picture with Larson's (1974) picture that elliptical galaxies might be formed through some slow dissipative process. Such models envisage the elliptical galaxy as composed of gas slowly dissipating its energy and 
turning itself into stars. Because of the dissipation, stars formed later and later form a denser and denser core. Thus, the envelope of the galaxy is built from the outside in. Larson (1974) has produced an excellent fit to the E1.5 galaxy NGC 3379, using a gaseous dissipation timescale $\tau_{\text {diss }}=1.19 t_{f}$, and a star formation timescale $\tau_{\text {star }}=$ $1.11 t_{f}\left(t_{f}=\right.$ free fall time), however, this model of NGC 3379 which achieves the best fit includes no infall.

There are several important factors that make one favor dissipationless infall models over dissipation models with no infall. (1) The observed flattening of ellipticals is predicted by the dissipationless theory, as we have discussed. It would seem that any slow dissipation process capable of producing an E5 galaxy could also produce an E8 galaxy. (2) We have argued strongly that infall must be present because the protogalaxy must be matched to a cosmological background solution. Infall is important both for galaxies in clusters and field galaxies. The present work shows that without dissipation infall produces an envelope in agreement with Hubble's Law, this would indicate that when infall is included, as it always should be, dissipation is unimportant. (3) Dissipation models must have $\tau_{\text {diss }} \sim t_{f}$ to work. For $\tau_{\text {diss }} \sim 0.1 t_{f}$ the models would be far too centrally condensed. The fact that spiral galaxies which contain appreciable amounts of gas are highly flattened, argues that gas, when it is present, is indeed highly dissipative. A self-supporting cloud of hot gas of galactic mass $\left(10^{11} \mathfrak{M}_{\odot}\right)$ and galactic dimensions $(10 \mathrm{kpc})$ has a bremsstrahlung cooling time an order of magnitude smaller than the free fall time. The gas density in such a gaseous protogalaxy is similar to the present gas density in the solar neighborhood. If the gas is composed of cold clouds with large translational velocities then the cloud-cloud collision time will be much shorter than a dynamical time if clouds in the protogalaxy have properties similar to those observed in the interstellar medium today. For example, clouds currently travel only $10^{7} \mathrm{yr}$ between collisions as compared with the dynamical time of $10^{8}$ yr (see Spitzer, 1968). These considerations suggest that $\tau_{\text {diss }} \lesssim 0.1 t_{f}$ is more realistic for gaseous dissipation than $\tau_{\text {diss }} \sim t_{f}$.

Let us now discuss the effects of supernovae in sweeping gas out of the galaxy (cf. Mathews and Baker, 1971). It is this effect that prevents elliptical galaxies formed via the dissipationless process from forming disks due to mass loss from evolved stars. Mathews and Baker (1971) show that in the central regions the gas density can be high enough to provide sufficient cooling to overbalance the supernovae and allow trapping and dissipation. Such a mechanism may indeed allow reprocessing of material in the very innermost regions and give rise to the cyanogen gradients in the nuclei of elliptical galaxies, observed by Spinrad et al. (1972). Alternatively, Tremaine et al. (1974) have proposed a mechanism for forming dense galactic nuclei from old globular clusters that have spiraled in toward the center through dynamical friction. The existence of many compact galactic nuclei (even in M31 and M32), which seem to be completely independent dynamical entities, is a warning that they may require special mechanisms for formation. Thus, the existence of cyanogen gradients in the very innermost regions of elliptical galaxies should not be used to infer that reprocessing and dissipation are important in the formation of the galaxy as a whole. 
To repeat, dissipationless collapse and relaxation models have a number of strong arguments supporting them. (1) The intrinsic flattenings of elliptical galaxies (E0 to E5 with just a few E6's) are in excellent agreement with that predicted from dissipationless collapse with simple virial theorem arguments. (2) Relaxed isophotes and velocity distributions can be produced naturally with the Lynden-Bell violent relaxation process. (3) With infall included, as it must be, elliptical galaxies are found to have envelopes in good agreement with Hubble's Law. (4) A detailed model including infall and tidal interactions with neighboring protogalaxies produces an excellent fit to the observed rotating galaxy NGC 4697. (5) With the Peebles' (1971) tidal interaction picture, and the present theory of dissipationless collapse, it is possible to understand why elliptical galaxies have such similar radial structures and yet exhibit a variety of runs of ellipticity versus radius. The radial structure is determined primarily by infall and tidal effects which are similar for all galaxies, but the run of ellipticity versus radius depends heavily on the shape of the original protogalaxy which varies from galaxy to galaxy. (6) Finally, the dissipationless collapse model for ellipticals provides a natural scenario for the production of elliptical and spiral galaxies as distinct classes. When star formation is essentially complete before the point of maximum collapse, a dissipationless collapse and relaxation occurs leaving an elliptical galaxy with flattening E0 to $\sim$ E5. Such a galaxy will show stars at all later times with little or no gas because gas from mass loss of stars will be swept out continually by supernovae. On the other hand, if significant gas is left over at the point of maximum collapse, it, being highly dissipative, will quickly form a flat disk. Thus, spiral galaxies should have both spheroidal and disk components, representing the material processed into stars before the collapse and that which was not. Once a galaxy has an appreciable disk, cooling will be sufficient to prevent supernovae from disrupting it and it will accumulate additional mass from stellar mass loss in the spheroidal component. This view is in complete agreement with the work of Ostriker and Peebles (1973) on the dynamics of spiral galaxies and with the work of Ostriker and Thuan (1974) on metal abundances in spiral galaxies.

All in all, the dissipationless collapse picture explains many of the observed features of elliptical galaxies and provides a natural explanation for the formation of elliptical and spiral galaxies as distinct types.

\section{Acknowledgements}

It is a pleasure to thank Drs Chris Wilson, Richard Larson, James E. Gunn, Ivan King, Gus Oemler, and Mr Paul Schechter for helpful conversations and comments. I especially thank Dr King for allowing use of his data prior to publication. This research was supported in part by the National Science Foundation [GP-36687X].

\section{References}

Aarseth, S. J.: 1973, Cited in Oemler, A. 1973.

Gott. J. R.. III and Gunn, J. E.: 1971, Astrophys. J. Letters 169, L13. 
Gott, J. R., III : 1973, Astrophys. J. 186, 481.

Gott, J. R., III, Gunn, J. E., Schramm, D. N., and Tinsley, B. M.: 1974, Astrophys. J. (in press).

Gott, J. R. III: 1975, submitted for publication.

Gunn, J. E. and Gott, J. R. III: 1972, Astrophys. J. 176, 1.

Hénon, M.: 1964, Ann. Astrophys. 27, 83.

Hubble, E.: 1930, Astrophys. J. 71, 231.

King, I. R.: 1974, private communication.

Larson, R. B.: 1974, Monthly Notices Roy. Astron. Soc. 166, 585.

Lynden-Bell, D.: 1967, Monthly Notices Roy. Astron. Soc. 136, 101.

Mathews, W. G. and Baker, J. C.: 1971, Astrophys. J. 170, 241.

Oemler, A.: 1973, Ph.D. Thesis, California Inst. of Technology.

Ostriker, J. P. and Peebles, P. J. E.: 1973, Astrophys. J. 186, 467.

Ostriker, J. P. and Thuan, T. X.: 1974, in press.

Peebles, P. J. E. and Dicke, R. H.: 1968, Astrophys. J. 154, 891.

Peebles, P. J. E.: 1970, Astron. J. 75, 13.

Peebles, P. J. E.: 1971, Astron. Astrophys. 11, 377.

Peebles, P. J. E.: 1974, Astrophys. J. Letters 189, L51.

Press, W. H. and Schechter, P.: 1974, Astrophys. J. 187, 425.

Spinrad, H., Smith, H. E., and Taylor, D. J.: 1972, Astrophys. J. 175, 649.

Spitzer, L.: 1968, Diffuse Matter in Space, Wiley, New York.

Tremaine, S., Ostriker, J. P., and Spitzer, L.: 1974, in press.

Wilson, C. P.: 1973, Ph.D. Thesis, Univ. of California, Berkeley.

\section{DISCUSSION}

King: It seems to me that we, as practitioners of dynamics, are making good progress in understanding the reasons for the existence and nature of the different types of galaxies. Both the preceding speakers have distinguished elliptical galaxies from disks, however, according to whether some considerable part of the original material remains in gaseous form. I think that this a crucial question in the nature of galaxies, and that we should go home and tell our colleagues in other fields that the key to understanding the nature of galaxies is first to understand the process of star formation.

Larson: Do I understand correctly that everything that you have said applies only to the outer most half or two-thirds of the density profile of NGC 4697, and that your models say nothing about the formation of the core regions of elliptical galaxies? If so, your models do not explain everything with no free parameters.

Gott: My model is designed to follow the envelope well, but because of cost constraints does not give information about very small scales in the center. In my model the velocity distribution has become nearly isothermal in the inner regions so that an isothermal model for the center would be indicated. Such models like those of King give a very good fit to the center parts of Hubble's law. Thus, one would expect the central region of the model to also give a good fit to the observations if one followed into the center. When isothermal conditions are established by the violent relaxation one has always fit the central parts of elliptical galaxies well. The problem in the past has always been the shape of the envelope. Also when discussing the formation of dense galactic nuclei it must be remembered that there may be additional special mechanisms involved.

Lecar: How is your model tied to the cosmology? For example, do you require that you form galaxies before clusters of galaxies?

Gott: Galaxies are larger than the Jeans mass at recombination so they and larger structures such as clusters can form as density perturbations. In the present picture galaxies which we observe to have the shortest crossing times form first, then having clusters of galaxies forming from statistical aggregates of galaxies.

Bertola: Using your model can you fit also the De Vaucouleurs' law for the luminosity profile of ellipticals?

Gott: A comparison of Hubble's law and De Vaucouleurs' law shows that they are quite close. De Vaucouleurs' law has a slight curvature to it that one can also see in the model results. In the model one knows the total mass of the galaxy and this agrees to within $10 \%$ of the extrapolated mass based on De Vaucouleurs' law. So that would indicate that it was a good fit. 
Innanen: Do these models lead in a natural explanation to a higher velocity dispersion in the radial direction compared to the axial dispersion?

Gott: For the rotating model, because of the angular momentum picked up by stars in the envelope due to the tidal interaction, the random velocity distributions were roughly although not accurately isotropic.

Pismis: Obviously there still remains a free parameter - at least one - in order to decide the fate of a galaxy, that is its type. I have often wondered whether the total mass of a galaxy may not be an important parameter in the evolutionary path followed by the galaxy. We know little about fragmentation mechanisms. But it is conceivable that in some way the distribution law of the fragments may be related to the total mass of the system, and that the distribution of fragment sizes affects the process of evolution. 